\title{
Método conglomerado-análisis discriminante-análisis envolvente de datos para clasificar $y$ evaluar eficiencia empresarial *
}

\author{
Tomás José Fontalvo-Herrera \\ Profesor de tiempo completo titular, Universidad de Cartagena. Cartagena de Indias-Colombia \\ tfontalvoh@unicartagena.edu.co (1) https://orcid.org/0000-0003-4642-925।
}

\section{Efraín De La Hoz-Granadillo}

Investigador Senior. Profesor de tiempo completo. Universidad de Cartagena, Cartagena de Indias-Colombia edelahozg@unicartagena.edu.co (D) https://orcid.org/0000-0002-5196-813X

En esta investigación se desarrolla un método que integra herramientas de análisis multivariado con el objetivo de identificar perfiles característicos de las pequeñas y medianas empresas exportadoras pequeñas y evaluar su eficiencia empresarial, de manera que se apoyen procesos de mejora en sus resultados. Para lo anterior, se revisaron elementos teóricos asociados a la eficiencia empresarial y el cálculo estadístico multivariado, lo que permitió desarrollar una metodología que integra el análisis de conglomerados, análisis discriminante y análisis envolvente de datos para evaluar la eficiencia empresarial. Se analizaron 45 empresas pequeñas y medianas exportadoras de Cartagena-Colombia, en las que se identificaron 3 perfiles característicos con niveles promedio de eficiencia de $71,89 \%$ el uno, 70,93\% el dos y 51,25\% tres. El análisis discriminante mostró la pertinencia y relevancia de los perfiles identificados lográndose un 95,6\% de clasificación correcta del modelo discriminante. Se concluye que las herramientas de cálculo multivariado analizadas en esta investigación son significativas para clasificar y evaluar la eficiencia de grupos empresariales.

Palabras clave Eficiencia técnica; análisis envolvente de datos; conglomerado; análisis discriminante; perfil empresarial

\section{Conglomerate method-discriminant analysis-data envelopment analysis to classify and evaluate business efficient}

A B S T R A C T

This research develops a method that integrates multivariate analysis tools with the aim of identifying characteristic profiles of small and medium-sized small exporting companies and evaluating their business efficiency, so that improvement processes are supported in their results. For the above, theoretical elements associated with business efficiency and multivariate statistical calculation were reviewed, which develops a methodology that integrates cluster analysis, discriminant analysis and data envelopment analysis to evaluate business efficiency. 45 small and medium exporting companies from Cartagena-Colombia were analyzed, in which 3 characteristic profiles were

Recibido: 07/1 1/2019 Aceptado: 07/04/2020

* (http://creativecommons.org/licenses/by-nc-sa/4.0/) Published by Universidad Libre - Cali, Colombia.

Cómo citar este artículo: FONTALVO-HERRERA,Tomás José; DE LA HOZ-GRANADILLO, Efraín. Método conglomerado-análisis discriminante-análisis envolvente de datos para clasificar y evaluar eficiencia empresarial. En: Entramado. Julio - Diciembre, 2020. vol. I6, no. 2, p. 46-55. 
identified with average efficiency levels of $71.89 \%$ for one, $70.93 \%$ for two and $51.25 \%$ for three. The discriminant analysis selected the relevance and relevance of the identified profiles, registering $95.6 \%$ of correct classification of the discriminant model. It is concluded that the multivariate calculation tools analyzed in this research are analyzed to classify and evaluate the efficiency of business groups.

KEYWORDS

Technical efficiency; data envelopment analysis; conglomerate; discriminant analysis, company profile

Jel Classification

$\mathrm{Cl} 9, \mathrm{OI}, \mathrm{O} 32$

\section{Análise de cluster-discriminatória-análise de envelopes de dados para classificar e avaliar a eficiência do negócio}

RES U M O

Nesta investigação é desenvolvido um método que integra ferramentas de análise multivariada com o objectivo de identificar perfis característicos das pequenas e médias empresas exportadoras e avaliar a sua eficiência empresarial, de forma a apoiar os processos de melhoria dos seus resultados. Para o efeito, foram revistos elementos teóricos associados à eficiência empresarial e ao cálculo estatístico multivariado, o que permitiu desenvolver uma metodologia que integra análise de clusters, análise discriminante e análise envolvente de dados para avaliar a eficiência empresarial. Foram analisadas quarenta e cinco pequenas e médias empresas de exportação em Cartagena-Colômbia, identificando três perfis característicos com níveis médios de eficiência de 71,89\% para uma, 70,93\% para duas e 51,25\% para três. A análise discriminante mostrou a pertinência e relevância dos perfis identificados, atingindo 95,6\% da classificação correcta do modelo discriminante. Conclui-se que as ferramentas de cálculo multivariado analisadas nesta investigação são significativas para classificar e avaliar a eficiência dos grupos empresariais.

Palavras-chave Eficiência técnica; análise de envelope de dados; cluster; análise discriminante; perfil do negócio

Classificação JEL $\mathrm{Cl} 9, \mathrm{O} 11, \mathrm{O} 32$

\section{Introducción}

Acorde con la tendencia del comercio internacional, Colombia ha suscrito tratados y acuerdos de libre comercio con países como Israel, Panamá, Reino Unido, México, Estados Unidos, El Salvador, Guatemala, Honduras, Comunidad Andina (CAN) Países del Mercosur, Chile, Comunidad del Caribe (Caricom), Canadá, Cuba, Nicaragua, Asociación Europea de Libre Comercio (AELC), Unión Europea, Alianza del pacífico, Costa Rica y acuerdo de alcance parcial con Venezuela y la República de Corea (Mincomercio, 2020), con los cuales busca incentivar el intercambio comercial, facilitando en doble vía la exportación e importación de productos a la industria.

En concordancia con lo anterior, Cartagena - Colombia actualmente cuenta con uno de los puertos más importante del país, lo anterior ha constituido al puerto en una de los más importantes a nivel de exportaciones. Sin embargo, las condiciones de la ciudad, requieren una ciudad con empresas altamente competitivas, por lo que se requieren estudios e investigaciones permanentes de las organizaciones que generan la dinámica comercial de la ciudad. Como antecedentes significativos se han desarrollado estudios similares en otros contextos, como el de Fontalvo, De la Hoz y De la Hoz (2018) en PYMES exportadoras de la ciudad de Barranquilla-Colombia.

En este sentido es de especial interés analizar cómo se desarrolla la racionalidad técnica de los grupos empresariales que exportan. Lo cual implica la necesidad de estudiar y analizar la eficiencia de los conglomerados o grupo empresariales que generan la dinámica exportadora de la ciudad. Lo anterior posibilita definir características, estructuras, niveles de desempeño y eficiencia de los grupos empresariales, lo que coadyuvará a poder comprender el estado de los diferentes perfiles empresariales y en función de esto poder generar políticas públicas que contribuyan con su mejora e incentiven la actividad exportadora del sector. Esto requiere el uso de elementos cuantitativos que permitan analizar la pertinencia de grupos característicos para 
su estudio y toma de decisiones, lo que se constituye en un aporte valioso para el sector empresarial. De esta manera, se justifica y se establece la pertinencia de esta investigación y proporciona sentido a las preguntas problemas que se plantean a continuación. ¿Qué patrones permiten determinar grupos de conglomerados empresariales en el sector exportador de Cartagena considerando los rubros financieros? ¿De qué manera se pueden identificar las diferencias significativas de los conglomerados empresariales en el sector exportador de Cartagena? ¿Como determinar la eficiencia de las Pequeñas y Medianas Empresas-PYMES exportadoras de Cartagena-Colombia? ¿Como integrar los procesos de clasificación, evaluación y análisis de eficiencia empresarial en un método?

Las preguntas problemas anteriores generaron los siguientes objetivos de investigación: i) Establecer un análisis de conglomerado que permitiera identificar las características de los perfiles empresariales del sector. ii) Clasificar la pertenencia de los perfiles empresariales mediante Análisis discriminante. iii) Evaluar la eficiencia promedio de las empresas de cada conglomerado empresarial. Lo que en su integralidad permite establecer un método ConglomeradoAnálisis Discriminante-Análisis Envolvente de Datos-DEA (por sus siglas en ingles) para clasificar y evaluar perfiles de eficiencia en empresas. Lo anterior ha generado como propósito de esta investigación desarrollar un método de cálculo multivariado que permita clasificar grupos de empresas exportadoras de la ciudad de CartagenaColombia, así como establecer los criterios estadísticos para validar la pertenencia de los grupos empresariales. Con lo que finalmente se puedan evaluar la eficiencia de las empresas pertenecientes a los perfiles empresariales definidos previamente. Evaluación integral que permita a los directivos de estas organizaciones el análisis, compresión y toma de decisiones para la mejora operacional que se vea reflejada en los resultados de la organización y en la competitividad empresarial de ésta en su contexto.

En concordancia con esta investigación, las técnicas de cálculo multivariado proporcionan un marco teórico y conceptual relevante para el estudio del contexto empresarial. En este sentido, autores como Yang \& Trewn (2004) plantean su importancia en el análisis e interpretación de datos de varias variables para la toma de decisiones. Díaz et al. (2013) consideran una estrategia importante para el análisis de grandes cantidades de información en los sectores industrial y financiero. Por su parte otros autores como (De la Garza, Morales, \& González, 2013), conceptúan que el análisis estadístico multivariante corresponde al conjunto de técnicas o métodos estadísticos utilizados para el análisis e interpretación de grandes volúmenes de datos, a partir del procesamiento de un conjunto de variables teniendo en
Por lo anterior, en esta investigación se presenta un método que integra herramientas de cálculo multivariado como son el Análisis de Conglomerados, el Análisis Discriminante y el Análisis Envolvente de Datos - DEA, que permite identificar y evaluar la eficiencia de perfiles característicos de las pequeñas y medianas empresas exportadoras de Cartagena-Colombia, con lo cual se contribuye con una herramienta de análisis para los procesos de toma de decisiones y mejoramiento empresarial.

\section{Marco teórico}

Análisis de Conglomerado: Técnica del cálculo multivariado utilizada para identificar estructuras jerárquicas en la partición de grupo de observaciones y la reducción de variables en la dimensión de un problema objeto de estudio (Peña, 2002). Según Levy \& Varela (2005) el análisis de conglomerado proporciona clasificaciones o tipologías a partir de observaciones inicialmente no clasificadas, las cuales pueden ser utilizadas en investigaciones académicas o aplicadas para encontrar conjunto de datos similares entre sí. Diferentes investigadores han aplicado y demostrado la pertinencia del análisis de conglomerado en el análisis empresarial. De La Hoz \& López (2017) muestran la importancia y capacidad de esta técnica en identificar perfiles característicos del potencial exportador. En el proceso de conformación de grupos, se utilizan medidas de distancias y criterios de eslabonamiento para la clusterización de las observaciones en el que se evalúa su nivel similaridad o disimilaridad. Para la medida de distancia entre dos muestras $i, j$ se utiliza frecuentemente el coeficiente de Pearson (ecuación I) que representa la correlación de las muestras (De la Garza et al., 20l3).

$$
R_{i j}^{2}=\frac{\sum_{k=1}^{r}\left(X_{i k}-\bar{X}_{k}\right)\left(X_{j k}-\bar{X}_{k}\right)}{\left[\sum_{k=1}^{r}\left(X_{i k}-\bar{X}_{k}\right)^{2} \sum_{k=1}^{r}\left(X_{j k}-\bar{X}_{k}\right)^{2}\right]^{2}}
$$

Con $X_{i k}$ y $X_{j k}$ valores que toma la variable $k$ en las muestras $i$ y $j, r$ número de variables y $\bar{X}_{k}$ valor promedio de la variable $k$ tomando todas las muestras. Según Catena et al. (2003) en el proceso de clusterización para conformar grupos homogéneos frecuentemente se utiliza el método de la varianza mínima o enlace de Ward (W) que minimiza el incremento de la varianza (ecuación 2) (Peña, 2002):

$$
W=\sum_{g=1}^{G} \sum_{i=1}^{n_{g}}\left(X_{i g}-\overline{X_{g}}\right)\left(X_{i g}-\overline{X_{g}}\right)^{\prime}
$$


Con $X_{i g}$ valor que toma la variable en el individuo $i$ y grupo $g, \overline{X_{g}}$ promedio de la variable en el grupo $g, n_{g}$ total individuos en el grupo $g, G$ total de grupos. En la selección de grupos a unirse se utiliza la ecuación 3 :

$$
\min \frac{n_{a} n_{b}}{n_{a}+n_{b}}\left(\overline{X_{a}}-\overline{X_{b}}\right)^{\prime}\left(\overline{X_{a}}-\overline{X_{b}}\right)
$$

Con $n_{a}$ y $n_{b}$ total de individuos en los grupos $a$ y $b$ a fusionarse, $\overline{X_{a}}$ y $\overline{X_{b}}$ media de los grupos $a$ y $b$.

Análisis Discriminante: Esta técnica del cálculo multivariado, es utilizada para identificar si existen diferencias significativas entre $g$ grupos de una muestra a partir del análisis de los $n$ datos de un conjunto $p$ de variables, para explicar sus diferencias y establecer procesos de clasificación de nuevas observaciones (Díaz, et al., 20I3). Para el proceso de clasificación de observaciones nuevas, la técnica define funciones discriminantes $Y_{i}$, a partir de la combinación lineal de variables originales con $\vec{X}=\left(\mathrm{X} 1, \mathrm{X} 2, \ldots, \mathrm{X}_{p}\right)$ un vector con $p$ variables aleatorias del problema, de manera que se minimice la variación intra grupo y se maximice la variación extra grupo (ecuación 4).

$\boldsymbol{Y}_{\boldsymbol{i}}=\boldsymbol{\beta}_{\boldsymbol{i} \mathbf{0}}+\boldsymbol{\beta}_{\boldsymbol{i} \mathbf{1}} \boldsymbol{X}_{\mathbf{1}}+\cdots+\boldsymbol{\beta}_{\boldsymbol{i p}} \boldsymbol{X p}, \mathrm{i}=1, \ldots, \min \{\mathrm{p}, \mathrm{g}-1\}$

Con $\boldsymbol{\beta}_{i 0}$ una constante, $\boldsymbol{\beta}_{i j}$ ponderación discriminante de la variable independiente $X_{j}, j=1, \ldots, p$.

Análisis Envolvente de datos DEA: Charnes, Cooper, Rhodes (1978), fueron pioneros en el desarrollo conceptual del análisis envolvente de datos DEA, la cual es una herramienta que permite analizar la eficiencia de un grupo de unidades productivas que utilizan los mismos recursos para producir los mismos productos. Para lo cual establece una frontera eficiente de las unidades productivas objeto de estudio. Para lo anterior estos autores plantean importante establecer las variables de entrada para cada unidad empresarial (DMU), así como las variables de salida para cada unidad productiva que permitan la maximización del grupo empresarial, la cual depende de las restricciones de todo el grupo empresarial. Diversos autores, han analizado en distintos contextos el concepto de eficiencia empresarial a partir del análisis envolvente de datos, lo que ha permitido identificar y valorar variables y modelos para el estudio de la eficiencia de las organizaciones, tal como se evidencia en los resultados de (Araya, 2019; Armenta, Barreto y Prieto, 2012; Fontalvo, Mendoza,Visbal, 2015a; Campoverde, Romero, Borenstein, 2019; Zamora y Favila, 2018). En esta investigación se utilizó el modelo CCR-O orientado a la maximización de las salidas (Chediak y Valencia, 2008). El modelo matemático utilizado para calcular la eficiencia está representado por la siguiente expresión:

Modelo DEA Básico (Modelo CCR - O): La eficiencia de los grupos objeto de estudio se evalúa por medio de la siguiente estructura matemática (ecuación 5 y 6):

$$
\begin{gathered}
\operatorname{Max} \mathrm{Z}=\frac{\sum_{\mathrm{r}=1}^{\mathrm{s}} \mathrm{u}_{\mathrm{ro}} \mathrm{y}_{\mathrm{ro}}}{\sum_{\mathrm{i}=1}^{\mathrm{m}} \mathrm{u}_{\mathrm{ro}} \mathrm{x}_{\mathrm{io}}} \\
\operatorname{Max} \mathrm{Z}=\frac{\sum_{\mathrm{r}=1}^{\mathrm{s}} \mathrm{u}_{\mathrm{rj}} \mathrm{y}_{\mathrm{rj}}}{\sum_{\mathrm{i}=1}^{\mathrm{m}} \mathrm{V}_{\mathrm{ij}} \mathrm{x}_{\mathrm{ij}}} \leq 1 ; \mathrm{j}=1, \ldots, \mathrm{n} \\
\mathrm{u}_{\mathrm{rj}} \geq 0, \quad \mathrm{~V}_{\mathrm{ij}} \geq 0 \\
\mathrm{r}=1, \ldots, \mathrm{s} \quad \mathrm{i}=1, \ldots, \mathrm{m}
\end{gathered}
$$

Siendo $\mathrm{u}_{\mathrm{ro}} \mathrm{y} \mathrm{x}_{\mathrm{io}}$ el conjunto de DMU más favorables, el anterior modelo se puede convertir en (ecuación 7):

$$
\begin{aligned}
& \operatorname{Max} \mathrm{Z}=\sum_{\mathrm{r}=1}^{\mathrm{s}} \mathrm{u}_{\mathrm{ro}} \mathrm{y}_{\mathrm{ro}} \\
& \sum_{1}^{\mathrm{m}} \mathrm{v}_{\mathrm{io}} \mathrm{x}_{\mathrm{io}}=1 \\
& \sum_{r=1}^{s} u_{r j} y_{r j} \leq \sum_{i=1}^{m} v_{i j} x_{i j} ;(j=1,2, \ldots, n) \\
& u_{r j} \geq 0, \quad v_{i j} \geq 0
\end{aligned}
$$

Siendo $n$ el número de DMU y $m$ es el número de variables de entrada y $s$ el número de variables de salida. Considerando lo anterior esta investigación tiene como propósito establecer un método que permita clasificar y evaluar perfiles de eficiencia financieros de las PYMES exportadoras de Cartagena, lo cual sea replicable en cualquier otro contexto.

\section{Metodología}

En la presente investigación se utilizó el análisis racional apoyado en evidencias empíricas y teóricas para estructurar un método de clasificación y evaluación de perfiles de eficiencia de empresas exportadoras. Se trabajó con las Pymes exportadoras de Cartagena-Colombia. Con el fin de alcanzar lo anterior, se identificaron las variables del modelo a partir del cual se identificaron los perfiles de eficiencia financiera. Seguidamente se integraron las técnicas de Conglomerado, Análisis Discriminante, Análisis envolvente de datos que definen el método propuesto en esta investigación. El método propuesto implicó seleccionar el sector empresarial, identificar y seleccionar las variables para la investigación, aplicar la técnica de 
análisis conglomerados para definir los perfiles, validar los perfiles empresariales por medio de Análisis Discriminante, establecer el nivel de consolidación y desarrollo de los perfiles empresariales, evaluar los perfiles de eficiencia financiera, para finalmente determinara los ranking de eficiencia de los perfiles financieros calculados al sector empresarial objeto de esta investigación.

El desarrollo del método propuesto se inicia con la identificación y selección del sector de la Pymes exportadoras de la ciudad de Cartagena-Colombia, compuesto por 45 empresas, de las cuales se tomó información primaria de la Cámara de Comercio de Cartagena-Colombia correspondiente al año 2018. Las variables seleccionadas fueron, número de empleados, total de activos, total pasivos, patrimonio e ingresos.

Con la información anterior, se aplicó el análisis de conglomerado en el que los resultados experimentales y la evidencia empírica, muestran la pertinencia de los modelos de Ward y Pearson para la conformación de los conglomerados y del análisis para validar el nivel de discriminación de las variables analizadas en los clústeres empresariales del sector. Para valorar los resultados del análisis de conglomerados se desarrolló la técnica de análisis discriminante en el que se utilizó el método de inclusión por pasos y el método de distancia de Mahalanobis con cálculo de probabilidades previas según tamaño de grupo para evaluar la capacidad de discriminación de las variables en los perfiles identificados. Finalmente, se definieron los clústeres de eficiencia empresarial de las Pymes por medio de DEA ( con los cuales se determinaron el nivel de madurez de los perfiles y los rankings o categorías de eficiencia empresarial identificados en el análisis de conglomerado. En esta investigación se trabajó con el software Minitab I 6 para el análisis de conglomerado y el análisis discriminante, y el software DEA Solver Pro para el cálculo de las eficiencias.

\section{Resultados y discusión}

\section{I. Análisis de Conglomerados}

Con el fin de identificar los conglomerados objeto de estudio de las PYMES exportadoras de la ciudad de CartagenaColombia, se desarrolló un estudio de conglomerados en el que inicialmente se hizo el análisis de nivel de distancias, lo cual determinó 3 grupos o perfiles homogéneos intragrupo para las empresas estudiadas. Con el estudio de análisis de codo, se analiza los niveles de distancias y estudia el proceso de agrupamiento que identifica la distancia 4,5019 con 3 conglomerados como el indicado para agrupar las Pymes exportadoras de la ciudad de Cartagena. Seguidamente, se evaluaron distintas medidas de distancia y eslabonamiento. Los resultados empíricos mostraron el criterio de interrelación (Medida de agrupamiento) Ward y la medida de similitud o semejanza el coeficiente de Pearson como los más pertinentes para esta investigación.

La Tabla I muestra el número de Pymes clasificadas por conglomerado y la distancia promedio desde el centroide de las observaciones clasificadas en los conglomerados. El conglomerado I o Perfil I agrupa II pymes exportadoras, el conglomerado 2 o Perfil 2 agrupa 17 Pymes y el conglomerado 3 o perfil 3 agrupa 17 pymes. Así mismo, la Tabla muestra el nivel de homogeneidad de los datos al compararse con los resultados de las distancias entre centroides de los 3 clústeres.

Tabla I.

Distancias desde y entre los centroides por conglomerados
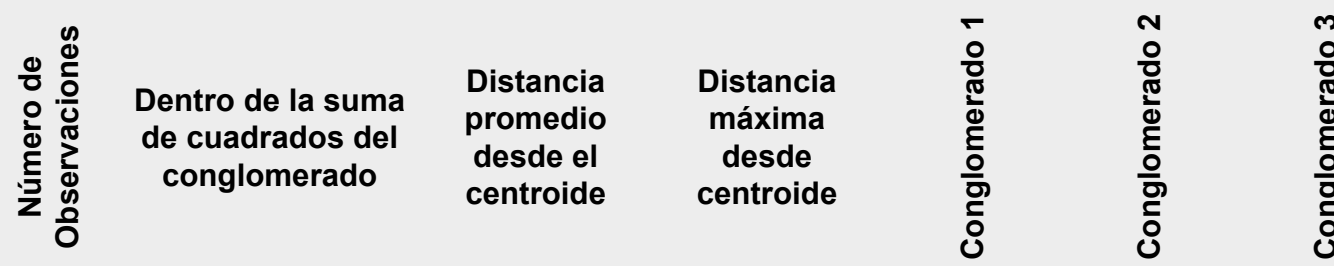

$\begin{array}{lccccccc}\text { Conglomerado1 } & 11 & 2,68 \mathrm{E}+21 & 1,17 \mathrm{E}+10 & 4,06 \mathrm{E}+10 & 0,00 \mathrm{E}+00 & 2,82 \mathrm{E}+10 & 2,22 \mathrm{E}+10 \\ \text { Conglomerado2 } & 17 & 2,22 \mathrm{E}+19 & 1,00 \mathrm{E}+09 & 2,38 \mathrm{E}+09 & 2,82 \mathrm{E}+10 & 0,00 \mathrm{E}+00 & 6,13 \mathrm{E}+09 \\ \text { Conglomerado3 } & 17 & 4,28 \mathrm{E}+20 & 4,48 \mathrm{E}+09 & 1,09 \mathrm{E}+10 & 2,22 \mathrm{E}+10 & 6,13 \mathrm{E}+09 & 0,00 \mathrm{E}+00\end{array}$

Fuente: Resultado de la investigación 
La Tabla 2, muestra los centroides de las variables por cada uno de los conglomerados, los resultados evidencian la heterogeneidad de los grupos o perfiles identificados, mediante la diferencia de los centroides de las variables en cada uno de los conglomerados. El dendograma de la Figura I, ilustra la conformación de los 3 grupos o perfiles empresariales. Las evidencias experimentales muestran la pertinencia de los tres clústeres para el estudio de las eficiencias de las empresas.
Al observar la Tabla 2, también se puede analizar que el perfil 3 es el grupo que mayores ingresos, patrimonio y activos posee, seguido en esto mismos rubros por el perfil I. El perfil que menos ingresos, patrimonio y activos genera es el perfil 2. De donde se puede evidenciar que la consolidación en el sector exportador de las empresas pequeñas y mediana depende de la fortaleza financiera del perfil financiero empresarial.

Tabla 2.

Centroides de grupo

\begin{tabular}{ccccc}
\hline Variable & Conglomerado1 & Conglomerado2 & Conglomerado3 & Centroide principal \\
\hline C1 & 9,850 & 9 & 18 & 34 \\
C2 & 15000000000 & 1043455451 & 4653025585 & 5827942839 \\
C3 & 10300000000 & 701812396 & 3116169302 & 3963173497 \\
C4 & 4730000000 & 341643055 & 1536856284 & 1864769342 \\
C5 & 23400000000 & 1258394755 & 5412699686 & 8242256461 \\
\hline
\end{tabular}

Fuente: Resultado de la investigación

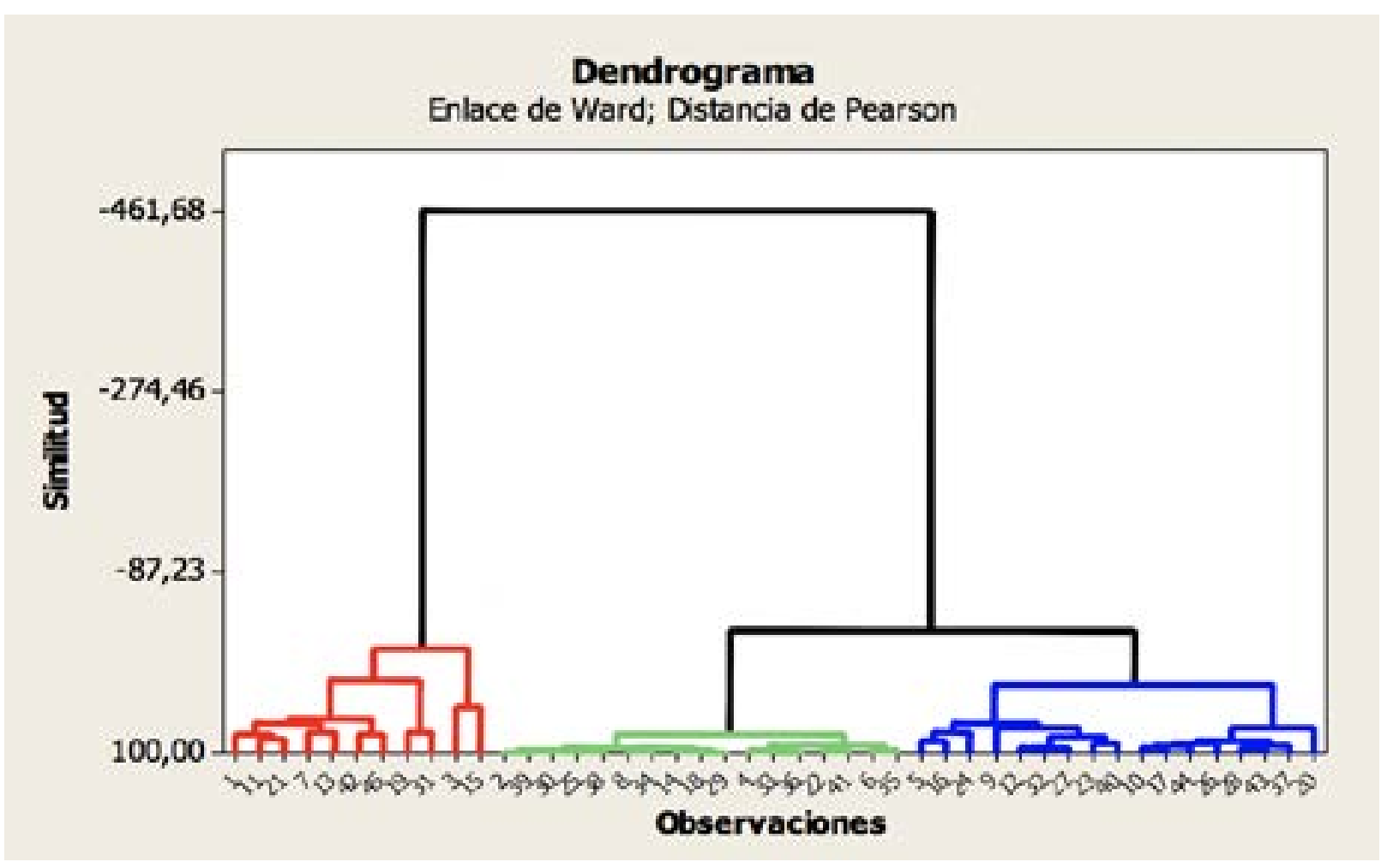

Figura I. Conformación de grupos o perfiles empresariales.

Fuente: Resultado de la investigación 


\subsection{Análisis Discriminante}

Seguidamente, se procedió a analizar la consistencia de los perfiles identificados en el análisis de conglomerados, para lo cual se realizó un análisis discriminante para evaluar la capacidad de clasificación correcta del modelo. La Tabla 3 muestra la matriz de confusión en donde se presentan los resultados del análisis en el que se logró un $100 \%$ de clasificación correcta para los perfiles I y 2, y $88,2 \% \%$ de clasificación correcta para el perfil 3 , lo que proporciona de manera general un 95,6\% de clasificación correcta del modelo discriminante, con lo que se valida la pertinencia y relevancia del modelo de clasificación con tres perfiles en las Pymes exportadoras de la ciudad de Cartagena-Colombia.

Evaluación de las eficiencias de los perfiles empresariales de las Pymes exportadoras de Cartagena Colombia:

Finalmente se evalúan las eficiencias promedio de los perfiles definidos previamente, optimizando las variables de salidas. De los cálculos realizados por medio de DEA para los tres perfiles definidos previamente, se pueden calcular las eficiencias para estos tres conglomerados o clúster. El perfil I obtuvo un desempeño de eficiencia de 71,89\%, el perfil 2 generó una eficiencia promedio de 70, $93 \%$ y el perfil 3 obtuvo una eficiencia promedio de $51,25 \%$. Lo anterior es significativo cuando se contrastan los resultados empresariales generados por otras investigaciones como las de Fontalvo et al (2015a) que evaluaron la eficiencia de las empresas de Barranquilla-Colombia, alcanzando una eficiencia de $56,62 \%$, Fontalvo (2014) que mostró un promedio de eficiencia de $31,85 \%$ del grupo empresarial analizado, Fontalvo et al (2018) analizaron empresas exportadoras de otra ciudad con eficiencia promedio del sector de $4 \mathrm{I}, 38 \%$ resultados muy por debajo a los alcanzado en esta investigación. Con todo anterior se tiene evidencia empírica para aseverar que los 3 perfiles evidencian buenos resultados en términos de eficiencia promedio.

Así mismo, en la Tabla 4 se observan los Ranking de los 3 conglomerados definidos en esta investigación. De donde se puede analizar que solo 3 empresas en el perfil I alcanzaron la eficiencia, 6 empresas del perfil empresarial 2 alcanzaron la eficiencia y 5 en el perfil 3 alcanzaron la eficiencia empresarial 3. Lo que muestra la necesidad de mejorar la toma de decisiones en las condiciones de operaciones de los diferentes perfiles, de tal manera que se mejore la eficiencia técnica y de esta manera pueda reflejarse mejores resultados en los estados financieros de las empresas objeto de estudio.

Los resultados alcanzados en este estudio son coherentes con los resultados de otra investigación como $\mathrm{De} \mathrm{La}$ Hoz, González y Santana (2016) en el que se analiza una metodología para medir perfiles competitivos del potencial exportador de las organizaciones empresariales. De igual manera, Crespo (20II) realiza un análisis discriminante para clasificar perfiles de riesgo financiero en instituciones pertenecientes al sistema financiero venezolano. Belmonte y Plaza (2008) evidenciaron resultados de eficiencia del 79,8\% y $89,7 \%$ en el contexto del sector cooperativo de España mediante aplicación de DEA. Otros autores como Perdomo \& Mendieta (2007); Quintanilha et al. (2012); Armenta et al. (20I2) han aplicado DEA para analizar la eficiencia en diversos contextos, evidenciando empíricamente la pertinencia del método.

Tabla 3.

Resultados de clasificación correcta por conglomerado

\begin{tabular}{|c|c|c|c|c|c|}
\hline & \multirow{2}{*}{ Conglomerado } & \multicolumn{3}{|c|}{ Predicción grupo de pertenecía } & \multirow{2}{*}{ Total } \\
\hline & & 1 & 2 & 3 & \\
\hline \multirow[b]{4}{*}{ Original } & 1 & 11 & 0 & 0 & 11 \\
\hline & 2 & 0 & 17 & 0 & 17 \\
\hline & 3 & 0 & 2 & 15 & 17 \\
\hline & 1 & $100 \%$ & $0 \%$ & $0 \%$ & $100 \%$ \\
\hline \multirow[t]{2}{*}{ Porcentaje } & 2 & $0 \%$ & $100 \%$ & $0 \%$ & $100 \%$ \\
\hline & 3 & $0 \%$ & $11,8 \%$ & $88,2 \%$ & $100 \%$ \\
\hline
\end{tabular}

Fuente: Resultado de la investigación 
Tabla 4.

Ranking de eficiencia de los 3 perfiles empresariales y sus DMU

\begin{tabular}{|c|c|c|c|c|c|}
\hline Rank & $\begin{array}{l}\text { Ranking de eficiencia de } \\
\text { perfil } 1 \text { empresarial DMU }\end{array}$ & Score & Rank & $\begin{array}{c}\text { Ranking de eficiencia de perfil } 2 \\
\text { empresarial DMU }\end{array}$ & Score \\
\hline 1 & DMU11 & 1 & 13 & DMU213 & 0,513583 \\
\hline 1 & DMU12 & 1 & 14 & DMU214 & 0,418264 \\
\hline 1 & DMU13 & 1 & 15 & DMU215 & 0,412374 \\
\hline 4 & DMU14 & 0,851962 & 16 & DMU216 & 0,360272 \\
\hline 5 & DMU15 & 0,837481 & 17 & DMU217 & 0,163098 \\
\hline 6 & DMU16 & 0,832175 & Rank & $\begin{array}{c}\text { Ranking de eficiencia de perfil } 3 \\
\text { empresarial DMU }\end{array}$ & Score \\
\hline 7 & DMU17 & 0,749849 & 1 & DMU31 & 1 \\
\hline 8 & DMU18 & 0,587748 & 1 & DMU32 & 1 \\
\hline 9 & DMU19 & 0,475446 & 1 & DMU33 & 1 \\
\hline 10 & DMU110 & 0,414781 & 1 & DMU34 & 1 \\
\hline 11 & DMU111 & 0,15865 & 1 & DMU35 & 1 \\
\hline Rank & $\begin{array}{l}\text { Ranking de eficiencia de } \\
\text { perfil } 2 \text { empresarial DMU }\end{array}$ & Score & 6 & DMU36 & 0,559591 \\
\hline 1 & DMU21 & 1 & 7 & DMU37 & 0,491473 \\
\hline 1 & DMU22 & 1 & 8 & DMU38 & 0,481972 \\
\hline 1 & DMU23 & 1 & 9 & DMU39 & 0,453167 \\
\hline 1 & DMU24 & 1 & 10 & DMU310 & 0,446858 \\
\hline 1 & DMU25 & 1 & 11 & DMU311 & 0,359401 \\
\hline 1 & DMU26 & 1 & 12 & DMU312 & 0,230208 \\
\hline 7 & DMU27 & 0,788121 & 13 & DMU313 & 0,180581 \\
\hline 8 & DMU28 & 0,773116 & 14 & DMU314 & 0,180079 \\
\hline 9 & DMU29 & 0,765514 & 15 & DMU315 & 0,140536 \\
\hline 10 & DMU210 & 0,704272 & 16 & DMU316 & 0,128097 \\
\hline 11 & DMU211 & 0,6134 & 17 & DMU317 & $6,20 \mathrm{E}-02$ \\
\hline 12 & DMU212 & 0,546223 & & & \\
\hline
\end{tabular}

Fuente: Resultado de la investigación

\section{Conclusiones}

De los resultados generados por esta investigación asociados con la evidencia empírica se, puede concluir que el método propuestos fue pertinente para definir 3 perfiles empresariales en el sector exportador de la ciudad de Cartagena. El modelo de clasificación utilizado para establecer el método arrojó una capacidad de clasificación de 95,6\% para los tres perfiles empresariales analizados, lo cual es muy bueno cuando se compara con resultados de otras investigaciones. Adicionalmente se puede concluir que el promedio de eficiencia de las empresas exportadoras es superior a otras empresas del sector, así como al de otros contextos empresariales como se evidencia previamente con los estudios comparativos. Lo anterior género como aporte importante la caracterización y evaluación de 3 perfiles empresariales, lo que a la postre se constituye en un referente competitivo e insumo para la toma de decisiones empresariales por parte de los directivos de estas empresas. Así como para diseñar y establecer políticas públicas 
que respondan a las necesidades de estos microempresarios, con lo que se estimule e incentive la actividad exportadora de las PYMES en la ciudad de Cartagena-Colombia.

Finalmente se puede concluir, de las características analizadas previamente, de los 3 clústeres empresariales financieros analizados, que estos guardan una relación directamente proporcional a los activos y patrimonio de estos perfiles empresariales, como se puede observar de la Tabla 2.

Por otro lado, esta investigación generó a la comunidad científica y al sector empresarial a nivel local, nacional e internacional un método que integra la aplicación de diferentes técnicas de análisis multivariante. Así como una herramienta de DEA que permitió clasificar grupos empresariales y evaluar la eficiencia de estos. Con lo que se facilita a la dirección de estas empresas criterios y herramientas que le permiten la toma de decisiones para la mejora de la eficiencia técnica de estas empresas.

Como futuras investigaciones se invita la comunidad científica e investigadora a replicar la metodología en otros contextos y sectores, que permita evaluar los resultados de eficiencia y desempeño de los perfiles de eficiencia financiera alcanzados en esta investigación. Esta investigación tiene como limitación, la aplicación de un único criterio de optimización (CCR-O), por lo cual, no se contempla otras condiciones de eficiencia que pueden alcanzarse.

\section{Conflicto de intereses}

El manuscrito fue preparado y revisado con la participación de todos los autores, quienes declaramos que no existe conflicto de intereses que ponga en riesgo la validez de los resultados presentados.

\section{Referencias bibliográficas}

I. ARAYA SOLANO, Manuel. Eficiencia competitiva de los cantones en Costa Rica: análisis del índice de competitividad cantonal basado en modelos frontera no-paramétricos. En:Tec Empresarial. 2019. vol. I3, no.3, p.78-92. https://dx.doi.org//0.18845/te.v/3i3.4599

2. ARMENTA-VERGARA, Rosa María; BARRETO-NIETO, Carlos Alberto; PRIETO-BUSTOS, William Orlando. Medición de la eficiencia en el uso de las regalías petroleras, una aplicación del análisis envolvente de datos. En: Rev. Finanzas y Política Económica. Enerojunio 2012. vol. 4, no.I, p.I3-32. https://repository.ucatolica.edu.col handle/10983/1052

3. BELMONTE UREÑA, Luis Jesús; PLAZA ÚBEDA, Jose Antonio. Análisis de la eficiencia en las cooperativas de crédito en España. datos (DEA). En: CIRIEC-España, Rev. Economía Pública, Social y Cooperativa. 2008. vol. 63, p. II3-133. https://www.redalyc.org/ pdf/I74//74/2307005.pdf

4. CAMPOVERDE CAMPOVERDE; Jorge Arturo; ROMERO GALARZA, Carlos Armando; BORENSTEIN, Denis. Evaluación de eficiencia de cooperativas de ahorro y crédito en Ecuador: aplicación del modelo Análisis Envolvente de Datos DEA. En: Contaduría y administración. 2019.vol.64, no. I, p.I-19.https://dx.doi.org/10.2220I/ fca.24488410e.2018.1449

5. CHARNES, Abraham; COOPER, Willian W.; RHODES, Eduardo L. Measuring the efficiency of decision-making units. In: European J. Operational Research. November, 1978. vol. 2 no.6, p.429-444. https://doi.org/10.1016/0377-2217(78)90138-8

6. CHEDIAK P., Francisco; VALENCIA A.; Luz Stella. Metodología para medir la eficiencia mediante la técnica del análisis envolvente de datos - DEA'. En: Rev.Vector. 2008. vol. 3, no. I, p.70-8I. http://vector.ucaldas. edu.co/downloads/Vector3 7.pdf

7. CATENA MARATINEZ, Andrés; TRUJILLO MENDOZA, Humberto Manuel; RAMOS ALVAREZ, Manuel Miguel. Análisis multivariado. Un manual para investigadores. Primera edición. Madrid: Editorial Biblioteca Nueva. ISBN: 84-9742-I I5-9. 2003. p. 120-160

8. CRESPO, Jesús Yoel. CAMEL vs. discriminante, un análisis de riesgo al sistema financiero venezolano. En: Ecos de Economía. 201 I. vol.33, p.25-47. http://www.scielo.org.co/pdf/ecos/v|5n33/vl5n33a2.pdf

9. DE LA GARZA GARCÍA, Jorge; MORALES SERRANO, Blanca Nieves; GONZÁLEZ CAVAZOS, Beatriz Adriana. Análisis Estadístico Mutivariante. Un enfoque teórico y práctico. México: Editorial Mc. Graw Hill. ISBN:978-507-I5-08I7-I. 20I3. p. I50-I78.

10. DE LA HOZ GRANADILLO, Efrain; LÓPEZ POLO, Ludys. Aplicación de técnicas de análisis de conglomerados y redes neuronales artificiales en la evaluación del potencial exportador de una empresa. En: Información Tecnológica. 2017.vol. 28, no.4, p.67-74. https://dx.doi. org//0.4067/S07/8-07642017000400009

II. DE LA HOZ GRANADILLO, Efraín; GONZÁLEZ, Ángel León; SANTANA R., Alfredo. Metodología de medición del potencial exportador de las organizaciones empresariales. En: Información Tecnológica. 2016. vol. 27, no.6, p.II-I8. https://dx.doi.org//0.4067/ S0718-07642016000600003

12. DíAZ RODRÍGUEZ, Martín; GONZÁLEZ, Ángel León; HENAO PEREZ, Alvin; DÍAZ MORA, Martín Emilio. Introducción al análisis estadístico multivariado. Barranquilla: Editorial Universidad del Norte. ISBN: 978-958-74I-358-8. 2013. p. 70-100.

13. FONTALVO, Tomás; DE LA HOZ, Efraín; DE LA HOZ, Enrique. Método análisis envolvente de datos y redes neuronales en la evaluación y predicción de la eficiencia técnica de pequeñas empresas exportadoras. En: Información Tecnológica. 2018. vol. 29, no.6, p.267276. https://dx.doi.org//0.4067/S07/8-07642018000600267

14. FONTALVO HERRERA, Tomás; MENDOZA MENDOZA, Adel; VISBAL CADAVID, Delimiro. Análisis comparativo de eficiencia 
financiera: estudio de un caso del sector BASC en Barranquilla. En: Prospectiva. 20I5. vol.13, no.2, p.16-24. https://dx.doi.org/10.15665/ rp.v| $3 \mathrm{i} 2.483$

15. FONTALVO HERRERA, Tomás José; MENDOZA MENDOZA, Adel; VISBAL CADAVID, Delimiro. Eficiencia en los procesos logísticos en las empresas certificadas en BASC Medellín mediante análisis envolvente de datos. En: U.D.C.A Act.\& Div. Cient. 2015. voll7, no.I. p.265-274. http://www.scielo.org.co/scielo.php?script=sci arttext\&pid=SO $23-42262014000100029 \& \mid n g=e n$

16. FONTALVO, Tomás. Aplicación de análisis discriminante para evaluar la productividad como resultado de la certificación BASC en las empresas de la ciudad de Cartagena. En: Contaduría y administración. 2014. vol.59, no.I, p.43-62. https://doi.org/10.1016/ S0I86-1042(14)7|243-4

17. LEVY MANGIN, Jean Pierre; VARELA MALLOU, Jesús. Análisis multivariable para las ciencias sociales, la ed. Editorial Pearson Educación. ISBN; 84-205-3727-6. Madrid-España. 2003. 420p.

18. MINCOMERCIO. Acuerdos vigentes. Bogotá. 2020. http://www.tlc. gov.co/acuerdos/vigente

19. PEÑA, Daniel.Análisis de datos multivariantes. Editorial Mc.Graw Hill, Madrid, España. 2002. p. 137-235.

20. PERDOMO, Jorge Andrés; MENDIETA, Juan Carlos. Factores que afectan la eficiencia técnica y asignativa en el sector cafetero colombiano: una aplicación con análisis envolvente de datos. En: Desarrollo y Sociedad. 2007. no. 60, p.3-45. http://www.scielo.org.col scielo.php?script $=$ sci_arttext\&pid $=$ SO I 20-35842007000200002\&lng $=$ en\&tlng=es

21. QUINTANILHA DA SILVEIRA, Juliana; CORREIA BAPTISTA SOARES DE MELLO, João Carlos; ANGULO-MEZA, Lidia. Evaluación de la eficiencia de las compañías aéreas brasileñas a través de un modelo híbrido de análisis envolvente de datos (DEA) y programación lineal multiobjetivo. En: Ingeniare. Revista Chilena de Ingeniería. 2012. vol.20, no.3, p.33I-342. http://dx.doi.org//0.4067/S07/833052012000300007

22. YANG, Kai;TREWN, Jayant. Multivariate statistical methods in quality management. New York: Mc.Graw Hill. ISBN: 978007I432085. 2004. p.I-8

23. ZAMORA TORRES, América Ivonne; FAVILA TELLO, Antonio. Medición de la eficiencia de la Innovación 2013-2016 mediante el Análisis Envolvente de Datos (AED) en red dinámica. En: Economía, sociedad y territorio, 2018. vol. 18, no.57, p.557-584. https://dx.doi. org/10.22136/est20181184 